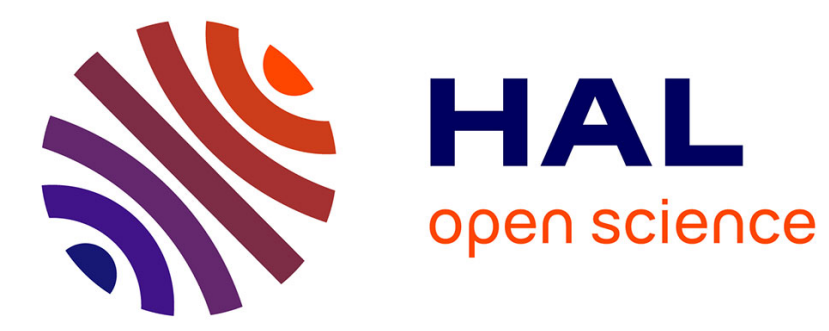

\title{
Parametric study of radiative heat transfer in participating gas-solid flows
}

Cyril Caliot, Vincent Eymet, Mouna El-Hafi, Yannick Le Maoult, G. Flamant

\section{To cite this version:}

Cyril Caliot, Vincent Eymet, Mouna El-Hafi, Yannick Le Maoult, G. Flamant. Parametric study of radiative heat transfer in participating gas-solid flows. International Journal of Thermal Sciences, 2008, 47 (11), pp.1413-1421. 10.1016/j.ijthermalsci.2007.10.016 . hal-01712152

HAL Id: hal-01712152

https://hal.science/hal-01712152

Submitted on 8 Nov 2018

HAL is a multi-disciplinary open access archive for the deposit and dissemination of scientific research documents, whether they are published or not. The documents may come from teaching and research institutions in France or abroad, or from public or private research centers.
L'archive ouverte pluridisciplinaire HAL, est destinée au dépôt et à la diffusion de documents scientifiques de niveau recherche, publiés ou non, émanant des établissements d'enseignement et de recherche français ou étrangers, des laboratoires publics ou privés. 


\title{
Parametric study of radiative heat transfer in participating gas-solid flows
}

\author{
C. Caliot ${ }^{\mathrm{a}, *}$, V. Eymet ${ }^{\mathrm{b}}$, M. El Hafi ${ }^{\mathrm{c}}$, Y. Le Maoult ${ }^{\mathrm{d}}$, G. Flamant $^{\mathrm{a}}$ \\ a Laboratoire Procédés, Matériaux et Energie Solaire, CNRS, 66120 Odeillo-Font-Romeu, France \\ b Laboratoire des Plasmas et Conversion de l'Energie, Université Paul Sabatier, 31000 Toulouse, France \\ c Centre de Recherches d'Albi en génie des Procédés des Solides Divisés, de l'Energie et de l'Environnement, Ecole des Mines d'Albi-Carmaux, 81000 Albi, France \\ ${ }^{\mathrm{d}}$ Centre de Recherche Outillages, Matériaux et Procédés, Ecole des Mines d'Albi-Carmaux, 81000 Albi, France
}

\begin{abstract}
Generally, before building a radiative model for a particular gas-solid flow, one has to determine whether the influence of particle scattering and gas radiation are significant. This paper gives the results of parametric studies on the influence of scattering and gas radiation for various gas-solid slabs at high temperature. A statistical Monte-Carlo method has been used in order to solve the radiative transfers into an emitting, absorbing and anisotropically scattering one-dimensional medium. These slabs are constituted of $\mathrm{H}_{2} \mathrm{O}$ an $\mathrm{dCO}_{2}$ gases with a monodispersion of spherical particles. In a first step, Planck means of particle radiative properties of absorption and scattering have been studied at high temperature versus the diameter $\left(10^{-7}<D<2 \cdot 10^{-4} \mathrm{~m}\right)$ and the complex refractive index $\left(n=1.7\right.$ o r2 5 an d1 $\left.\sigma^{4}<k<20\right)$. Then, fluxes emitted by numerous slabs have been computed and compared to emphasize the role of gases and particles. These fluxes are emitted by slabs with different thicknesses $(e=0.1 \mathrm{~m}$ or $1 \mathrm{~m})$, volume fraction $\left(10^{-7}<f_{v}<10^{-3}\right)$ and refractive index. In addition, particular attention is devoted to the use of the presented sensitivity results. An approach is then derived which permits to extend these results to other situations than those considered in this paper. Finally, the influence of the gas mixture and scattering on spectral emission is studied spectrally.
\end{abstract}

Keywords: Monte Carlo; Non-gray gases; Spherical particles; Anisotropic scattering; Refractive index

\section{Introduction}

Thermal radiation, in high temperature gas-solid flows, is considered to play a significant role with respect to the other heat transfer mode. Two-phase mixture is common in engineering practice, e.g. particle-gas heat exchanger, pulverized coal furnace, solid rocket motor etc. But the presence of various radiating gases, soot particles and polydispersed particulates complicates significantly the study of such processes. For example, combustion processes generate gaseous species, mostly $\mathrm{CO}_{2}$ and $\mathrm{H}_{2} \mathrm{O}$, and particles (soot, fly-ash particles or carbon aggregates).

In such situations, modelling of thermal radiation is far from being complete due to many complexities such as radiative properties of non-gray gases, multi-dimensional geometry

\footnotetext{
* Corresponding author.

E-mail address: cyril.caliot@promes.cnrs.fr (C. Caliot).
}

and computational accuracy as well as efficiency. In addition particles absorb, emit and scatter anisotropically thermal radiation. Their radiative properties are strongly dependent on composition, which affects their optical properties. Moreover, the particle size distributions and the volume fraction affect the extinction coefficient, the scattering albedo and the angular distribution of the scattered intensity of the particulate phase. For these reasons, modelling radiative transfer in a high temperature gas-solid flow is computer time consuming. To reduce this computation time, the prediction of the radiant exchange uses various methods, models and assumptions depending on the nature of the problem to solve. A better understanding of the major radiative processes involved in the studied application can be reached by performing a sensitivity analysis. As an example, in order to study potential simplifications in engineering applications of coal furnaces, isotropic and neglecting of scattering are evaluated against anisotropic scattering in [1]. Furthermore, isotropic and anisotropic scattering heat transfer processes have been analyzed in a parametric study [2]. Another parametric 
study was performed in [3] to examine the non-gray radiative effects of the two-phase mixture in an axisymmetric cylinder by varying parameters such as the temperature of particles, the non-gray gas composition and the particle concentration.

At the beginning of a survey and before building a radiative model for a gas-solid flow, one must have identified the main radiative transfer contributions involved. Then, respective contributions of gas and particles to the whole emission and the influence of scattering have to be evaluated. Gas spectra have a fine structure that is produced by line transitions. Consequently, the spectrum has to be divided into narrow bands and the treatment of the radiative transfer involves great resources in computer time and memory. For particles, the multi-scattering processes complicate the radiative model and require additional computing resources. From that point of view, it is recommended to construct a radiative transfer model in a gas-solid flow after firstly estimating the role of gas emission/absorption and particles scattering.

This paper aims to present a sensitivity study which permits, on the one hand, to evaluate the influence of scattering on the radiant exchanges in a particular gas-solid flow and, on the other hand, to assess the influence of gas emission/absorption in such a flow. For this purpose, a Monte-Carlo method is used to compute the radiant heat flux leaving a one-dimensional homogeneous layer containing non-gray gases $\left(\mathrm{H}_{2} \mathrm{O}\right.$ and $\left.\mathrm{CO}_{2}\right)$ and a monodispersion of spherical particles which scatter anisotropically. Since it is difficult to measure the optical properties of particles in a two-phase flow, this paper addresses this difficulty by considering a wide range of refractive index and by performing a sensitivity analysis.

In this paper, the radiative transfer model using the MonteCarlo method and the CK spectral model is presented first. Secondly, the methods for the computation of the radiative properties of gases and particles are given. Then, a sensitivity study is reported to show the dependency of particle radiative properties on their optical properties. Moreover, the influence of scattering by particles and gases radiation on the spectrum emitted by the layer is emphasized.

\section{Description of the radiative transfer model and the radiative properties}

To reach a better understanding of the influence of scattering as well as the influence of gas emission/absorption in a realistic gas-solid flow, an approach is proposed, in this paper, which consists to realize parametric studies on simplified situations.

The first simplification addresses the 3D geometry involved in realistic problems. The complex 3D geometry of a specific application can be simplified to a 3D slab (1D geometry). This simplification is chosen to consider only one geometrical parameter: the layer thickness $e$ (Fig. 1); which is characteristic of the realistic problem. Obviously, the thicknesses of the two other dimensions are infinite. Then, the influence of both scattering and gas contribution on the emitted flux by the layer ( $\Phi$ in Fig. 1) is maximized since optical thicknesses are maximum in the perpendicular directions of the slab thickness $e$. The second simplification addresses the distribution, into the

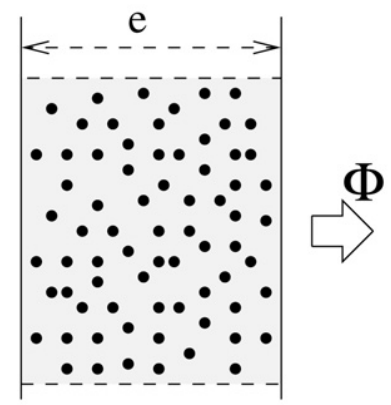

Fig. 1. Schematic representation of the total radiative flux $\Phi$, leaving the 1D layer composed of $\mathrm{H}_{2} \mathrm{O}, \mathrm{CO}_{2}$ gases and a homogeneous monodispersion of spherical particles, both at thermal equilibrium.

slab, of particles and gases concentrations, as well as temperature. In this study, a homogeneous slab with constant concentrations and temperature is considered. This simplification is done to perform reference sensitivity studies of several homogeneous media which are intended to be compared to realistic and non-homogeneous media. Then, the sensitivity results give one some information about the influence of scattering and gas radiation on the emitted flux by a realistic media. But, prior to the use of the sensitivity results, one has to decide how to use them. If the situation is close to a homogeneous one, the sensitivity results deliver quantitative informations. But, if the homogeneous assumption is far from being realistic, one can divide the medium into layers and apply the sensitivity results to find quantitative informations for each homogeneous layer.

In the following, the study deals with analysis of the emitted radiation from a $1 \mathrm{D}$ homogeneous layer containing a two-phase mixture (Fig. 1). The layer contains $\mathrm{H}_{2} \mathrm{O}$ and $\mathrm{CO}_{2}$ gases with a monodispersion of spherical particles at high temperature. The computation of emitted fluxes by the 1D layer is achieved by a Monte-Carlo Method (MCM) allowing one to solve radiative transfer including phenomena of emission, absorption and anisotropic scattering.

The Monte-Carlo simulation code that was used in the present paper is named KARINE. ${ }^{1}$ The underlying algorithm is presented in [4]; its convergence qualities have been improved on the basis of methodological developments that consisted in both a reformulation of radiative transfer in terms of Net Exchanges [5-8] and an optimization of sampling laws $[6,9,10]$. These efforts permitted to solve the well known convergence problem of traditional Monte-Carlo algorithms in the case of strong optical thickness configurations [11]. The formulation used in [11] has been generalized in order to take into account the scattering phenomena. The algorithm is fully $3 \mathrm{D}$, but it is used in KARINE for simulations of radiative transfer in monodimensional configurations [12], such as planetary atmospheres.

This algorithm allow one to compute the total flux emitted by the homogeneous slab. Furthermore, the algorithm takes into account the emission and absorption of the two-phase mixture

\footnotetext{
1 http://www.energetique.ups-tlse.fr/ eymet/karine.html.
} 
along a 3D multi-scattering path with the length $\ell$. The total flux emitted by the two-phase layer can be written as:

$$
\begin{aligned}
\Phi= & \frac{1}{2} \int_{0}^{\infty} \mathrm{d} \lambda \int_{V} \mathrm{~d} V \int_{4 \pi} \mathrm{d} \Omega(\vec{s}) \int_{\Gamma(V, S)} P_{\Gamma}(\gamma) \mathrm{d} \gamma \kappa_{\lambda} \\
& \times \exp \left[-\kappa_{\lambda} \ell_{\gamma}\right] L_{b \lambda}(T)
\end{aligned}
$$

with $\mathrm{d} V$ an elementary volume in the layer and $\mathrm{d} \Omega$ the elementary solid angle in the $\vec{s}$ direction $(\mathrm{d} \Omega=\sin (\theta) \mathrm{d} \theta \mathrm{d} \phi$ with $\theta$ and $\phi$ respectively the altitudinal and azimuthal angle). $\kappa_{\lambda}$ is the total monochromatic absorption coefficient (particles and gases) and $L_{b \lambda}$ is the monochromatic black body intensity at the temperature $T$ of the layer. One can notice that the definition of $\mathrm{d} V$ and $\kappa_{\lambda}$ implies there is enough particles in $\mathrm{d} V$ to assume the radiative properties to be homogeneous. $\Gamma(V, S)$ is the whole optical paths emitted by $V$ and reaching the $S$ surface (layer's border). $\gamma$ is the variable of the optical path including multi-scattering over all $\Gamma$ possibles $(\gamma \in \Gamma) . P$ is the probability density function of appearance of $\gamma$. Every multi-scattering paths are computed with a random step model. $\ell_{\gamma}$ is the length of the optical path with multi-scattering.

The spectral model used for the two-phase mixture is a $k$-distribution model with the Correlated-K (CK) assumption $[13,14]$. The spectral treatment with the MCM algorithm is detailed in [4]. A Gauss-Legendre quadrature of 10 points is chosen for the CK method.

Regarding gases, radiative properties are obtained directly from high resolution spectra computed by a line-by-line method over the spectral range $\left(800-8000 \mathrm{~cm}^{-1}\right)$. This spectral range is chosen since we consider that it covers the significant part of gaseous spectra at high temperature. Line-by-line spectra are computed from spectroscopic parameters of $\mathrm{CO}_{2}$ and $\mathrm{H}_{2} \mathrm{O}$ respectively included in CDSD1000 [15] and in the ONERA database [16]. The line by line model and the method employed to compute $\mathrm{CK}$ parameters for the gas mixture are presented by the present authors in [17]. Then, the CK parameters are found for the mixture of $\mathrm{H}_{2} \mathrm{O}$ and $\mathrm{CO}_{2}(\mathrm{CK}$ model with the single mixture gas assumption, [17]). In addition, the $\mathrm{CK}$ method does not produce any error on the emitted flux by the slab due to the correlation problem, since the slab is homogeneous.

Assuming particles as spherical, their radiative properties are computed following the Lorentz-Mie theory [18], in the spectral range (800-25000 $\left.\mathrm{cm}^{-1}\right)$. Absorption and scattering coefficients of particles, respectively $\kappa_{p}$ and $\sigma_{p}$, are then obtained in addition with the phase function, $p$, and the asymmetry factor $g$. The MCM algorithm needs the asymmetry factor of particles to compute the smooth phase function of Henyey-Greenstein, $p_{\mathrm{HG}}$, in order to optimise random samples and save computer time:

$p_{\mathrm{HG}}(\mu)=\frac{1}{4 \pi} \frac{1-g^{2}}{\left[1+g^{2}-2 g \mu\right]^{3 / 2}}$

$g=\int_{-1}^{1} \mu p(\mu) \mathrm{d} \mu$

with $\mu$ the cosine of the scattering angle. Radiative properties of particles are considered constant in a narrow band. These radia- tive properties depend on the spectral complex refractive index, $m_{\lambda}=n_{\lambda}-\mathrm{i} k_{\lambda}$, of particles (refractive index of gases is unity, $m_{\lambda}=n_{\lambda}=1$ ), the wavelength $\lambda$ of the incident beam, the diameter $D$ and the volume fraction $f_{v}$ of particles. Considering a monodispersion of spherical particles, absorption and scattering coefficients, respectively $\kappa_{p \lambda}$ and $\sigma_{p \lambda}$, are defined by:

$\kappa_{p \lambda}=\frac{3}{2} f_{v} \frac{Q_{a \lambda}(m, D)}{D}$

$\sigma_{p \lambda}=\frac{3}{2} f_{v} \frac{Q_{s \lambda}(m, D)}{D}$

where, $Q_{a \lambda}$ and $Q_{s \lambda}$ are respectively the spectral absorption and scattering efficiency which are computed following the Lorentz-Mie theory. In this paper, absorption and scattering coefficients are computed for a wide range of the parameters such as $f_{v}, m(n=\Re\{m\}, k=\Im\{m\}), D$ and $\lambda$. Radiative properties of particles are computed for a wide range of the particle diameter, $10^{-7}<D<2.10^{-4} \mathrm{~m}$. The volume fraction $f_{v}$ is varying between, a weak value encountered in applications, $10^{-7}$, and a large value, $10^{-3}$, which is below the limit volume fraction where both dependent absorption and dependent scattering occur significantly (limit $f_{v}=6.10^{-3}$ [19]). For convenience, the complex refractive index is considered constant over the spectrum. Two values of the real part of the complex refractive index is chosen, $n=1.7$ and $n=2.5$. Numerous particles have their refractive index, $n$, between this two values of $\Re\{m\}$. The particles with $n=1.7$ are typically metal oxide and the particles with $n=2.5$ are carbon particles. Furthermore, the imaginary part of the refractive index is varying, $10^{-4}<k<20$, to address the effects of slight changes in particle composition which affect the optical properties of particles.

Two parametric studies are presented in the paper. A sensitivity study on the radiative properties of particles is detailed first. Secondly, the influence of scattering and gas radiation on the radiant flux emitted by various $1 \mathrm{D}$ layers is presented.

\section{Sensitivity studies}

As described in the previous section, the Lorentz-Mie theory is applied to compute the radiative properties of particles. The corresponding results are analysed in Section 3.1. The Monte-Carlo algorithm is then used to compare the radiant fluxes emitted by scattering and non-scattering 1D layers (Section 3.2). In the same manner, the Monte-Carlo algorithm is used in Section 3.3 to determine the influence of gas radiation. In Section 3.4, a discussion and additional results are provided to extend the findings of the study to other configurations. Finally, the influence of gases and scattering on spectra will be shown in Section 3.5.

\subsection{Radiative properties of particles}

Emission, absorption and scattering of light by a small spherical particle depend on diameter, wavelength and refractive index. In the following, radiative properties of a particle are studied on the basis of a sensitivity analysis that uses diameter and 


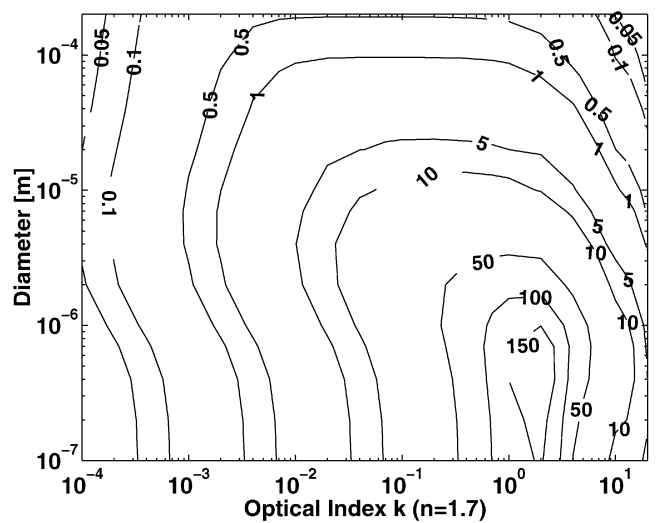

(1) $n=1.7, T=1000 \mathrm{~K}$.

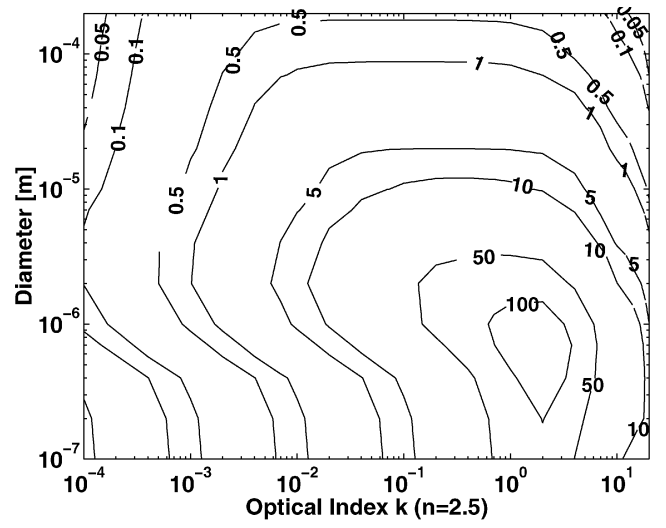

(3) $n=2.5, T=1000 \mathrm{~K}$.

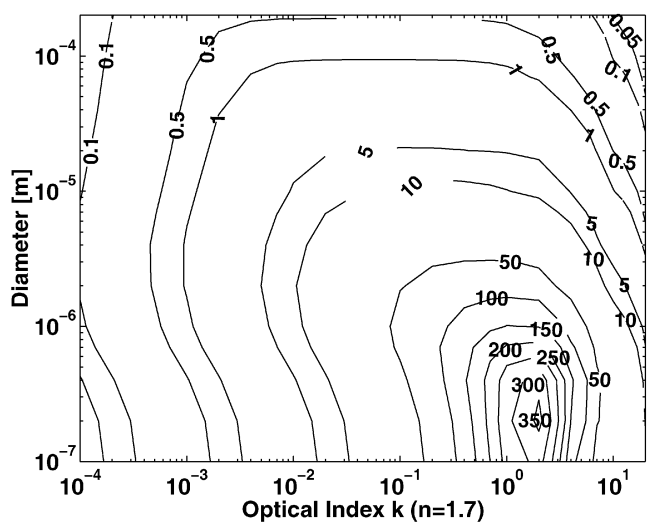

(2) $n=1.7, T=2000 \mathrm{~K}$.

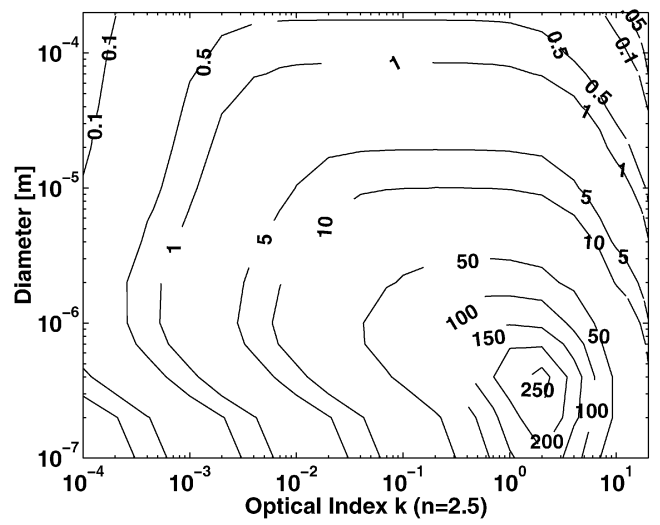

(4) $n=2.5, T=2000 \mathrm{~K}$.

Fig. 2. Mappings of $\frac{Q_{a}^{*}}{D} 10^{-4}\left[\mathrm{~m}^{-1}\right]$ versus the particle diameter and the optical index $k(m=n-\mathrm{i} k)$, for two values of $(n=1.7,2.5)$, and for two values of the particle temperature, $1000 \mathrm{~K}$ and $2000 \mathrm{~K}$.

refractive index as parameters. In order to compare several radiative properties of particles at high temperature, the spectral behaviour of their properties is taken into account by considering average quantity over the spectrum. Average values of the absorption and scattering coefficient (from Eqs. (4) and (5)) are then obtained, in the case of a homogeneous monodispersion, with:

$$
\begin{aligned}
\kappa_{p}^{*} & =\frac{\int_{0}^{\infty} \kappa_{p \lambda} L_{b \lambda}(T) \mathrm{d} \lambda}{\int_{0}^{\infty} L_{b \lambda}(T) \mathrm{d} \lambda}=\frac{3}{2} f_{v} \frac{1}{D} \frac{\int_{0}^{\infty} Q_{a \lambda} L_{b \lambda}(T) \mathrm{d} \lambda}{\int_{0}^{\infty} L_{b \lambda}(T) \mathrm{d} \lambda} \\
& =\frac{3}{2} f_{v} \frac{Q_{a}^{*}}{D}
\end{aligned}
$$

$$
\begin{aligned}
\sigma_{p}^{*} & =\frac{\int_{0}^{\infty} \sigma_{p \lambda} L_{b \lambda}(T) \mathrm{d} \lambda}{\int_{0}^{\infty} L_{b \lambda}(T) \mathrm{d} \lambda}=\frac{3}{2} f_{v} \frac{1}{D} \frac{\int_{0}^{\infty} Q_{s \lambda} L_{b \lambda}(T) \mathrm{d} \lambda}{\int_{0}^{\infty} L_{b \lambda}(T) \mathrm{d} \lambda} \\
& =\frac{3}{2} f_{v} \frac{Q_{s}^{*}}{D}
\end{aligned}
$$

These mean values, denoted with a star (Eqs. (6) and (7)), depend on the particle temperature and also on the energy distribution over the spectrum described by the Planck's law. Mappings of $\frac{Q_{a}^{*}}{D}$ and $\frac{Q_{s}^{*}}{D}$ (unity $\mathrm{m}^{-1}$ ) versus the particle diameter and the optical index $k(\Im\{m\}, m=n-\mathrm{i} k)$ are shown respectively in Figs. 2 and 3.

Maps of $\frac{Q_{a}^{*}}{D}$, in Figs. 2.1 and 2.2, are obtained for $n=1.7$ and for temperature $T=1000 \mathrm{~K}$ and $T=2000 \mathrm{~K}$ respectively.
Although monochromatic radiative properties are identical for this two temperatures, Planck mean values are greater for $T=$ $2000 \mathrm{~K}$. Indeed, as the temperature increases and according to Wien's law, much importance is given to small wavelength by the Planck's law where the absorption coefficient is large. In Figs. 2.3 and 2.4 the same comment can be done for particles with $n=2.5$.

Considering the same particle temperature but with different refractive index, such as in Figs. 2.2 and 2.4, we find the maximum values of $\frac{Q_{a}^{*}}{D}$ of particles with $n=1.7$ to be higher than those of the particles having $n=2.5$. Maxima are reached for optical index values near unity $(0.1<k<10)$ [20] and for size parameter $x=\frac{\pi D}{\lambda}$ close to unity. As the Lorentz-Mie theory predicts [18], interactions between particles and light are maximum for $x=1$. At $2000 \mathrm{~K}$, significant fluxes are emitted in the range $(0.5-10 \mu \mathrm{m})$ and the diameter range, matching $x=1$, is about $(0.16-3 \mu \mathrm{m})$. This is the same interval of diameter we find in Figs. 2.2 and 2.4 where radiative properties are maximum.

Concerning $\frac{Q_{s}^{*}}{D}$ maps, they are shown in Fig. 3 and $\frac{Q_{s}^{*}}{D}$ values are also maximum for $x$ close to unity. The maximum values of $\frac{Q_{s}^{*}}{D}$ are higher for $n=2.5$ than for $n=1.7$. Furthermore, in the diameter range $(0.5-5 \mu \mathrm{m})$, where the scattering properties are maximum, a decrease in magnitude occurs for $k$ close to unity $(0.5<k<1)$. For the temperature dependence similar comments than those of the previous section can be given: the 


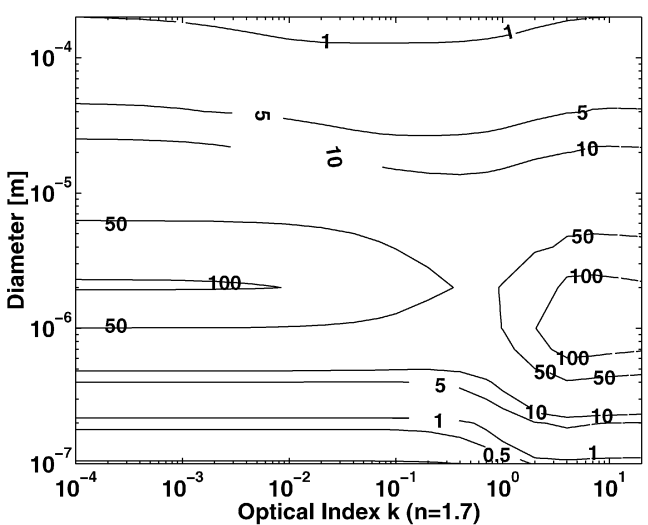

(1) $n=1.7, T=1000 \mathrm{~K}$.

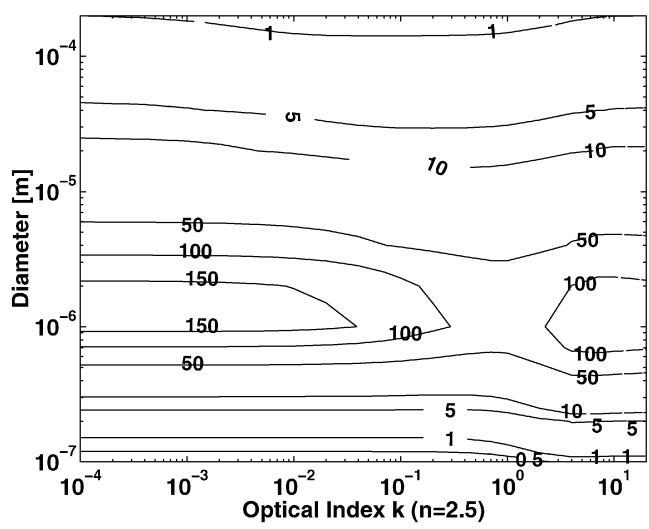

(3) $n=2.5, T=1000 \mathrm{~K}$

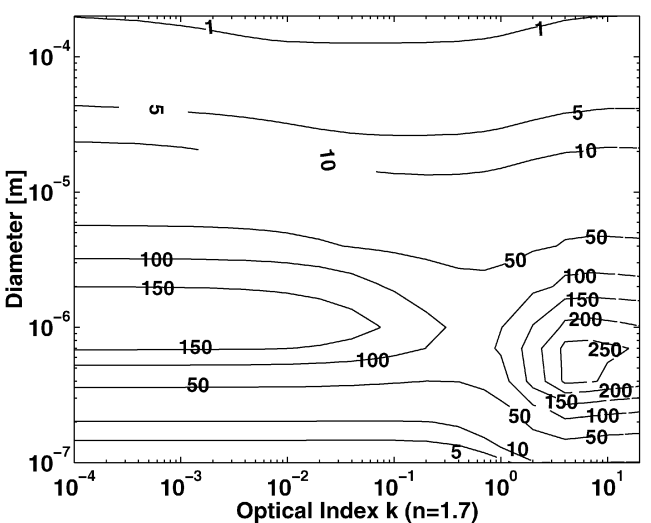

(2) $n=1.7, T=2000 \mathrm{~K}$

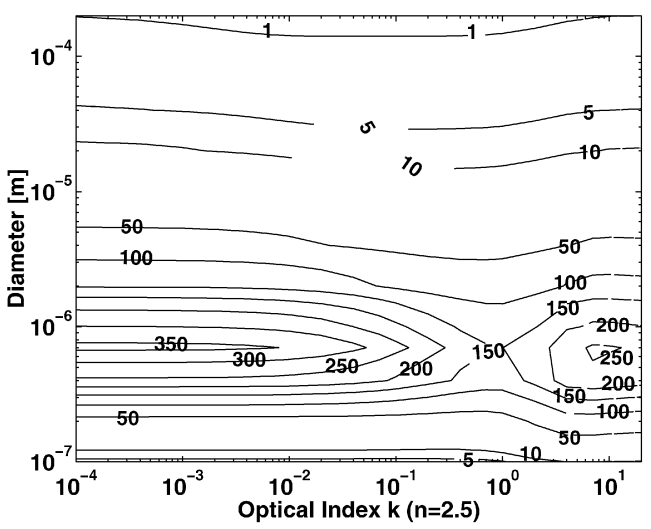

(4) $n=2.5, T=2000 \mathrm{~K}$.

Fig. 3. Mappings of $\frac{Q_{s}^{*}}{D} 10^{-4}\left[\mathrm{~m}^{-1}\right]$ versus the particle diameter and the optical index $k$, for two refractive indexes $(n=1.7,2.5)$, and for two values of the particle temperature.

maximum values of the averaged radiative properties are higher at $2000 \mathrm{~K}$ than at $1000 \mathrm{~K}$.

\subsection{Influence of scattering}

The influence of scattering is investigated in a parametric study on the radiative fluxes leaving a $1 \mathrm{D}$ homogeneous layer containing gases and particles, both at the same high temperature, $2000 \mathrm{~K}$ (Fig. 1). The gaseous phase contains non-gray $\mathrm{H}_{2} \mathrm{O}, \mathrm{CO}_{2}$ gases and a non-participating gas, $\mathrm{N}_{2}$, at atmospheric pressure $\left(10^{5} \mathrm{~Pa}\right)$. Molar fraction of the mixture are chosen representative of a fossil fuel combustion, such as $x_{\mathrm{H}_{2} \mathrm{O}}=0.2$, $x_{\mathrm{CO}_{2}}=0.1$ and $x_{\mathrm{N}_{2}}=0.7$. The monodispersion of spherical particles is composed of particles with $D=1 \mu \mathrm{m}$.

The influence of scattering is studied using a ratio $R_{S}$ which indicates the error due to neglecting scattering in the radiative transfer model:

$R_{s}=\frac{\Phi_{n s}-\Phi}{\Phi} 100$

where, $\Phi_{n s}$ is the total flux leaving the layer which is computed neglecting the scattering processes and $\Phi$ is the total flux taking into account scattering (Eq. (1)). Fig. 4 represents mappings of the $R_{S}$ ratio for particles with different refractive index, $n=1.7$ in Fig. 4.1, or $n=2.5$ in Fig. 4.2. The layer thickness is $e=$ $0.1 \mathrm{~m}$ (Fig. 1). The standard deviation of the statistical results
$\Phi$ and $\Phi_{n s}$, computed with the MCM algorithm, are both lower than $3 \%$.

Figs. 4.1 and 4.2 show maximum values of $R_{S}$ for high levels of volume fractions. As a matter of fact, influence of scattering is significant when the scattering optical thickness $\left(t_{s}^{*}=\sigma_{p}^{*} e\right)$ is large. One can notice the behaviour of $R_{S}$ with the optical index, $k$, that is close to the behaviour of $\frac{Q_{s}^{*}}{D}$ (Figs. 3.2 and 3.4, $D=1 \mu \mathrm{m})$. Nevertheless, for values of $k$ near unity, when absorption is great, $R_{S}$ values are minimum since absorption hide scattering processes. Indeed, as scattering increases the path length of photon bundles, their absorption along this multi-scattering path is increased as well. So, if absorption is large, non-scattered photon bundles or multi-scattered ones, are both attenuated and the emitted flux is no longer affected by scattering processes.

Following the comparison between Figs 4.1 and 4.2, $R_{S}$ ratios are greater for the layer containing particles with refractive index $n=2.5$ than the ones with $n=1.7$. Moreover, scattering influence appears to be smaller for particles with $n=1.7$ than for particles with $n=2.5$, specially when optical index $k$ is lower than $10^{-2}$. This is due to the influence of the scattering coefficient. Indeed, as mentioned in the study of $\frac{Q_{s}^{*}}{D}$, scattering for particles with $n=2.5$ is greater than scattering for particles with $n=1.7$. 


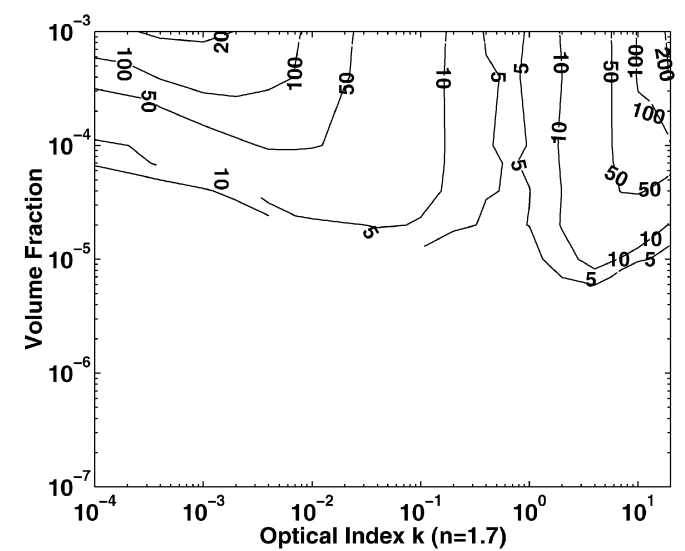

(1) $e=0.1 \mathrm{~m}, D=1 \mu \mathrm{m}, n=1.7$.

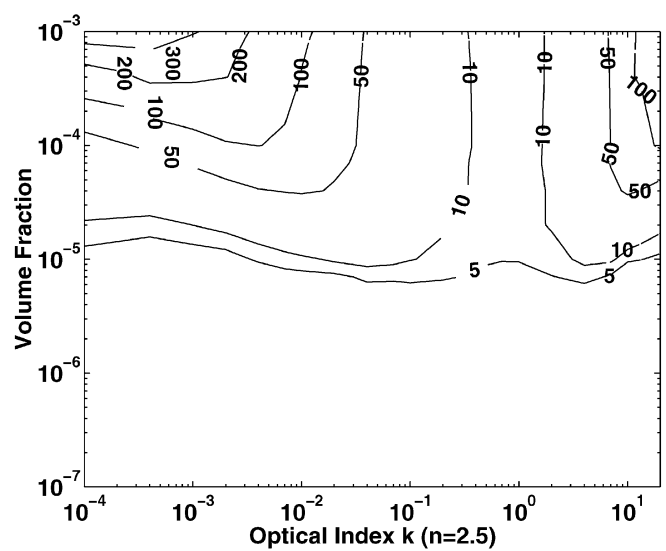

(2) $e=0.1 \mathrm{~m}, D=1 \mu \mathrm{m}, n=2.5$.

Fig. 4. Mappings of $R_{S}[\%]$ versus the volume fraction and the optical index $k$, for two values of the refractive index, $n=1.7,2.5(e=0.1 \mathrm{~m}, T=2000 \mathrm{~K})$.

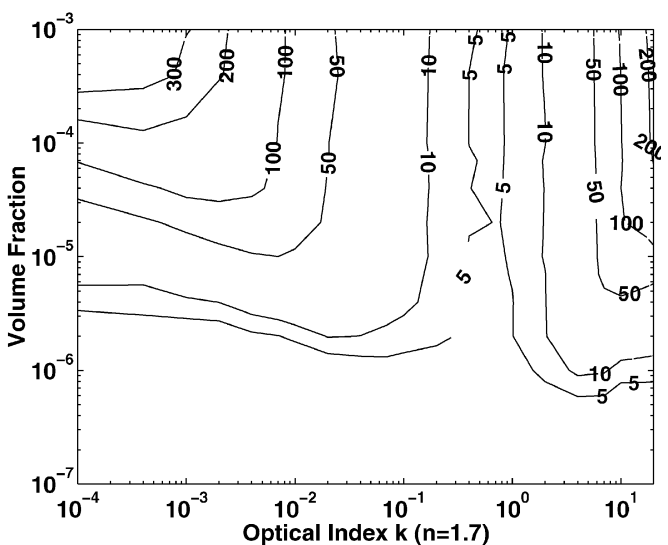

(1) $e=1 \mathrm{~m}, D=1 \mu \mathrm{m}, n=1.7$.

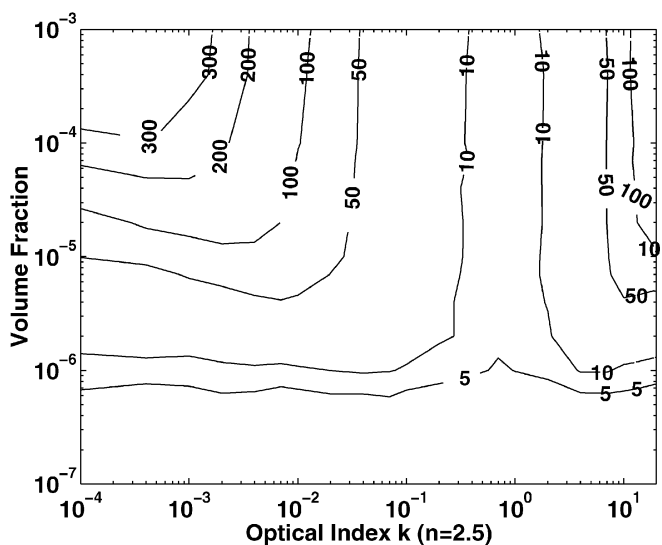

(2) $e=1 \mathrm{~m}, D=1 \mu \mathrm{m}, n=2.5$.

Fig. 5. Mappings of $R_{S}[\%]$ versus the volume fraction and the optical index $k$, for two values of the refractive index, $n=1.7,2.5(e=1 \mathrm{~m}, T=2000 \mathrm{~K})$.

Figs. 5.1 and 5.2 represent mappings of the $R_{S}$ ratio in the case of a $1 \mathrm{~m}$ thickness layer $(e=1 \mathrm{~m})$. Comments on these Figs. 5.1 and 5.2 are similar to the previous one since only the layer thickness is increased to 1 meter. Then, the scattering optical thickness of the layer is greater than the previous one $(e=0.1 \mathrm{~m})$ and the influence of scattering is enhanced. So, neglecting scattering is acceptable in layers with lower particle volume fractions.

In this section, sensitivity studies have shown, for the different test cases described before, that the influence of scattering can be neglected $\left(R_{S}<5 \%\right)$ for any values of refractive indexes if $f_{v}<6.10^{-6}$ in a $0.1 \mathrm{~m}$ thickness layer or $f_{v}<6.10^{-7}$ in a $1 \mathrm{~m}$ thickness layer.

\subsection{Influence of gases radiation}

The importance of gases radiation is represented by the use of mappings of the $R_{g}$ ratio:

$R_{g}=\frac{\Phi-\Phi_{p}}{\Phi} 100$

where, $\Phi_{p}$ is the total flux emitted by the layer containing only particles. The standard deviation of the statistical results $\Phi$ and $\Phi_{p}$, obtained by a Monte-Carlo simulation, are both lower than $3 \%$.

Fig. 6 shows maps of $R_{g}$ versus the volume fraction of particles $(D=1 \mu \mathrm{m})$ and the optical index $(k)$ for two values of the thickness layer. The real part of the refractive index is $n=1.7$, and the temperature of the mixture is $T=2000 \mathrm{~K}$. Maps in Fig. 6 indicate that gas radiation may be neglected only if the volume fraction is high enough and for $k$ values around unity $(0.1<k<10)$. One can notice the shape of the maps which is linked to the shape of $\frac{Q_{a}^{*}}{D}$ versus $k$ (Fig. 2.2, $D=1 \mu \mathrm{m}$ ). Indeed, when the absorption optical thickness is dominated by particle absorption, gas radiation is absorbed and the emission is principally due to particles. Concerning the results of $R_{g}$ ratio for layers with particle refractive index $n=2.5$, they are not represented since results are identical as those obtained for $n=1.7$.

This section treats the influence of gas radiation, and one can define the limit value of the $R_{g}$ ratio equal to $5 \%$ in order to neglect gas radiation. This limit is reached when the medium has a volume fraction $f_{v}>5.10^{-6}(e=0.1 \mathrm{~m})$ or $f_{v}>10^{-6}$ $(e=1 \mathrm{~m})$, both for $k$ values close to unity. In these regions of the $k-f_{v}$ domain gas radiation can then be neglected since particle absorption is dominant. For lower $k$ values both gas and particle play a significant role in the emission of the mixture. 


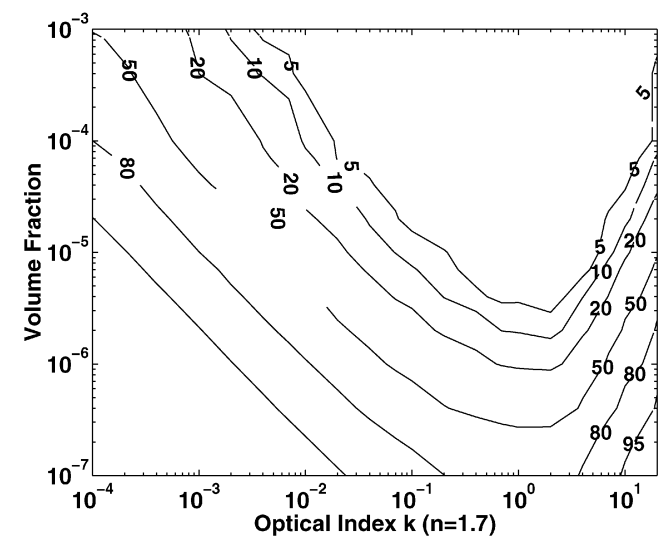

(1) $e=0.1 \mathrm{~m}, D=1 \mu \mathrm{m}, n=1.7$.

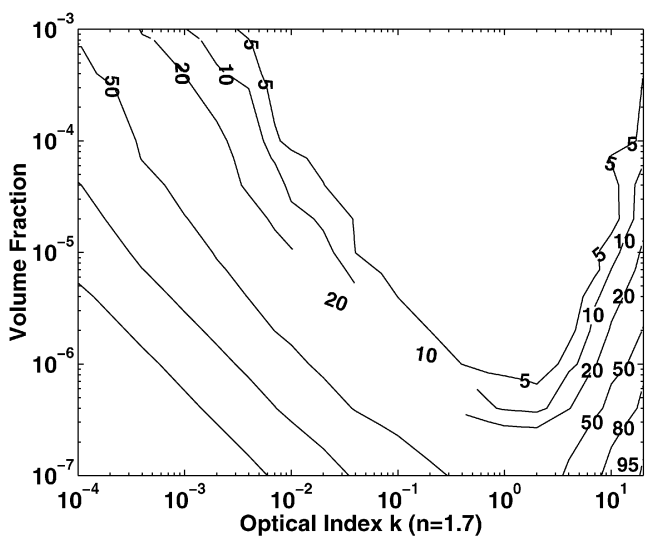

(2) $e=1 \mathrm{~m}, D=1 \mu \mathrm{m}, n=1.7$.

Fig. 6. Mappings of $R_{g}$ [\%] versus the volume fraction and the optical index $k$, for two values of the layer's thickness, $e=0.1 \mathrm{~m}$ and $e=1 \mathrm{~m}$, containing particles with refractive index, $n=1.7(T=2000 \mathrm{~K})$.

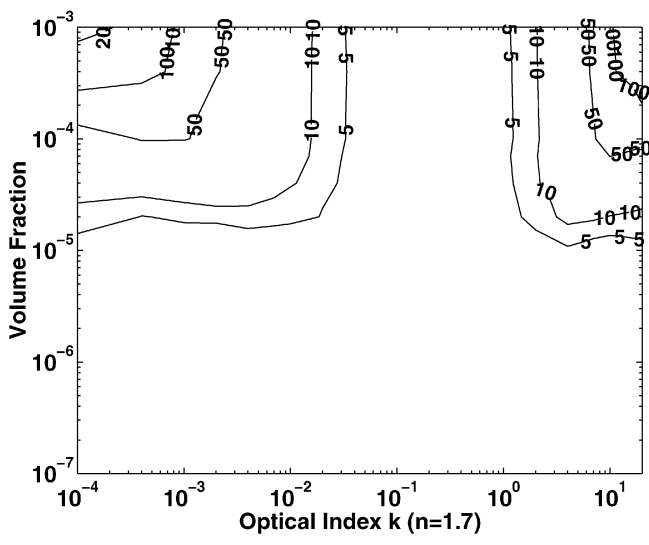

(1) $e=1 \mathrm{~m}, D=10 \mu \mathrm{m}, n=1.7$.

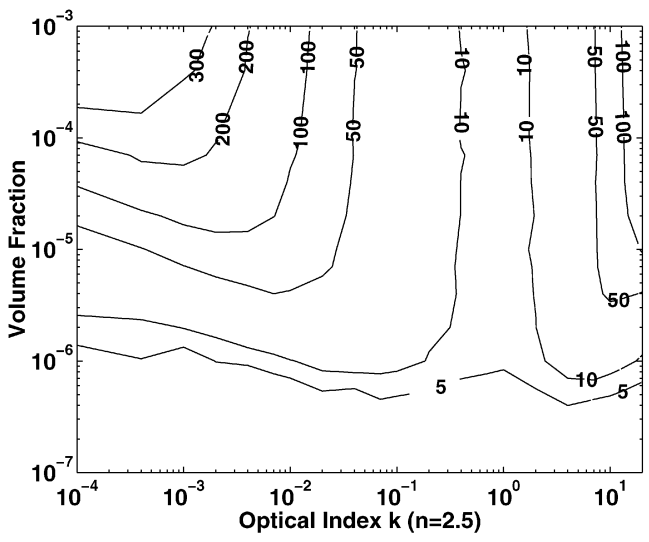

(2) $e=1 \mathrm{~m}, D=0.7 \mu \mathrm{m}, n=2.5$.

Fig. 7. Mappings of $R_{S}[\%]$ versus the volume fraction and the optical index $k$, for two slabs at $T=2000 \mathrm{~K}$.

\subsection{Extension of sensitivity results}

The extension of the sensitivity results to other situations can not be done quantitatively with the mappings presented in this paper. For such a purpose, other sensitivity studies involving specific parameters have to be considered. But, the present study should be helpful for qualitative studies of slabs involving temperature between $1000 \mathrm{~K}$ and $2000 \mathrm{~K}$ and particles with refractive index between 1.7 and 2.5. For example, let us consider two slabs at a temperature of $2000 \mathrm{~K}$ containing both gases and particles and having a thickness of $e=1 \mathrm{~m}$. They also contains the same concentrations of gases such as the ones given in the previous studies $\left(x_{\mathrm{H}_{2} \mathrm{O}}=0.2, x_{\mathrm{CO}_{2}}=0.1\right.$ and $\left.x_{\mathrm{N}_{2}}=0.7\right)$. The first slab, named Slab1, contains a monodispersion of particles with $D=10 \mu \mathrm{m}$ and $n=1.7$ and the second, named Slab2, contains particles with $D=0.7 \mu \mathrm{m}$ and $n=2.5$. The aim of this example is to show how to qualitatively use the sensitivity results given previously. Indeed, the prediction of the limit volume fraction should be done on the basis of a comparison between the particles radiative properties.

Concerning Slab1 $(D=10 \mu \mathrm{m}$ and $n=1.7)$, the radiative properties (Figs. 2.2 and 3.2) of its particles are weaker than those of particles with $D=1 \mu \mathrm{m}(n=1.7)$. Furthermore, sen- sitivity results for the slab with $D=1 \mu \mathrm{m}$ and $n=1.7$, yield to the following limit value of volume fraction: if $f_{v}<6 \times 10^{-7}$ (Fig. 5.1) scattering is negligible, for all values of $k$. Then, as a consequence of the qualitative approach, one can predict that the limit volume fraction required to neglect scattering, in Slab1, must be higher than $6 \times 10^{-7}$. Indeed, since the radiative properties of particles of Slab1 are weaker than those of particles with $D=1 \mu \mathrm{m}(n=1.7)$, the absorbed and scattered parts of the emitted flux in the Slab1 must be lower than the ones in the reference slab, $D=1 \mu \mathrm{m}(n=1.7)$ (Fig. 5.1). In order to give a comprehensive proof, the mapping of $R_{S}$ is computed and shown in Fig. 7.1. The qualitative approach is then verified, since the limit volume fraction for scattering is $10^{-5}$.

Regarding Slab2 ( $D=0.7 \mu \mathrm{m}$ and $n=2.5)$, the radiative properties of its particles are greater than those with $D=1 \mu \mathrm{m}$ (Figs. 2.4 and 3.4). Moreover, the sensitivity results of the reference slab ( $D=1 \mu \mathrm{m}$ and $n=2.5)$ show that scattering is negligible if $f_{v}<6 \times 10^{-7}$ (Fig. 5.2), for all $k$ values. Then, using the qualitative approach, one can predict that the limit volume fraction of Slab2 must be lower than $6 \times 10^{-7}$. As in the previous example, the mapping of $R_{S}$ is computed for Slab2 and the sensitivity results are shown in Fig. 7.2. These results 


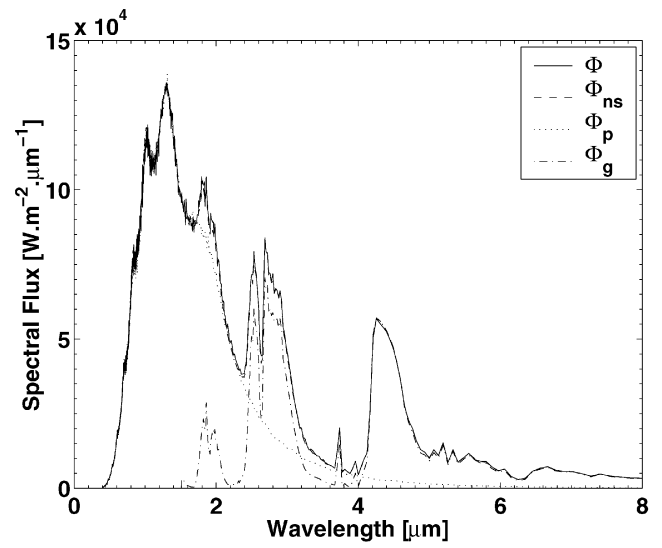

(1) $e=0.1 \mathrm{~m}, R_{S} \approx 0 \%, R_{g} \approx 34 \%$.

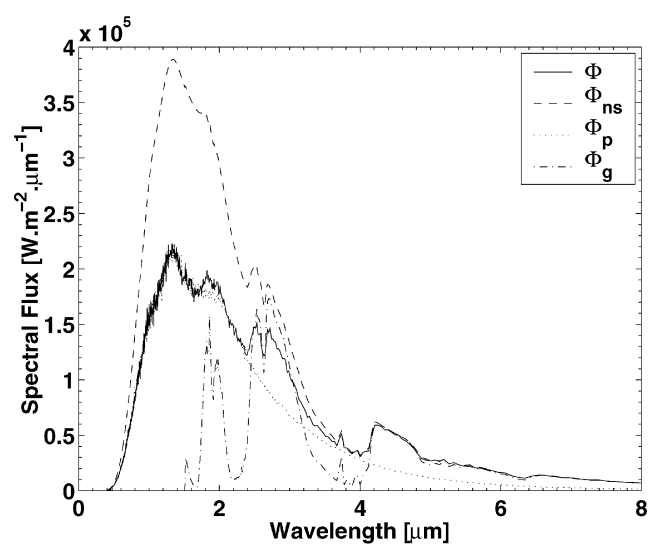

(2) $e=1 \mathrm{~m}, R_{S} \approx 47 \%, R_{g} \approx 21 \%$.

Fig. 8. Influence of scattering and gases radiation on spectral fluxes emitted by two layers having different thicknesses and same composition.

verify the qualitative approach since the limit value of the volume fraction, in the case of Slab2, is $4 \times 10^{-7}$.

\subsection{The spectral dependency}

Previous sections present discussions related to parametric analysis using average radiative properties over the spectrum. In this section we propose to evaluate the influence of gas radiation and scattering over the spectrum. For that purpose, spectral fluxes emitted by a 1D layer will be compared in Fig. 8. Then, Fig. 8 represents spectral fluxes leaving two slabs with different thicknesses ( $e=0.1 \mathrm{~m}$ and $e=1 \mathrm{~m}$ ) accounting for or neglecting radiation from gases and scattering from particles. Each layer (similarly to those presented previously) contains a gas mixture $\left(x_{\mathrm{H}_{2} \mathrm{O}}=0.2, x_{\mathrm{CO}_{2}}=0.1, x_{\mathrm{N}_{2}}=0.7\right)$ and a monodispersion of spherical particles $\left(D=10^{-6}, m=1.7-i \times 10^{-2}\right.$, $\left.f_{v}=10^{-5}\right)$ both at thermal equilibrium $(T=2000 \mathrm{~K})$ and atmospherical pressure $\left(10^{5} \mathrm{~Pa}\right)$.

Fig. 8.1 shows the spectral fluxes, $\Phi, \Phi_{n s}, \Phi_{p}, \Phi_{g}$ emitted by a one-dimensional slab where the thickness is fixed, $e=0.1\left(\Phi_{g}\right.$ is the flux emitted by a slab containing only the gas mixture). One can notice that scattering is negligible. Besides, the $R_{S}$ ratio is zero. But gases radiation is important, since $R_{g} \approx 34 \%$. We can observe the common spectral behaviour of the radiative species such as $\mathrm{H}_{2} \mathrm{O}$ and $\mathrm{CO}_{2}$. The absorption and emission bands of gases are located in the spectrum around $1.8 \mu \mathrm{m}\left(\mathrm{H}_{2} \mathrm{O}\right.$ band $), 2.5\left(\mathrm{H}_{2} \mathrm{O}\right.$ band $), 2.7\left(\mathrm{H}_{2} \mathrm{O}-\mathrm{CO}_{2}\right.$ bands), $4.3\left(\mathrm{CO}_{2}\right.$ band $)$ and beyond $\left(\mathrm{H}_{2} \mathrm{O}\right.$ band $)$.

Fig. 8.2 represents spectral fluxes for a slab when the thickness is increased, $e=1 \mathrm{~m}$. In this case, the influence of scattering is more important since $R_{S}$ is about $47 \%$. The major discrepancies due to scattering neglect arise between the wavelengths 0.5 and $4 \mu \mathrm{m}$ where scattering coefficient is important. Also, gases radiation has a significant contribution to total emission with a $R_{g}$ ratio reaching $21 \%$. But, particles scatter and, in the same time, absorb a large part of the radiation emitted by the gas mixture, as it can be seen when $\Phi_{g}$ and $\Phi$ are compared in Fig. 8.2. Then, the amount of radiation emitted by the gas mixture and the particles cloud inside the slabs are decreased by the scattering effects.

\section{Conclusion and future works}

The mean scattering and absorption radiative properties of spherical particles at $1000 \mathrm{~K}$ and $2000 \mathrm{~K}$ were computed for various particles diameter and imaginary part, $k$, of the refractive index, whose real part was chosen as $n=1.7$ and $n=2.5$. For the absorption, largest values are obtained in the diameter range $(0.1-20 \mu \mathrm{m})$ with $10^{-2}<k<2$. Moreover, the largest scattering radiative properties are found, for all $k$, in the same diameter range $(0.1-20 \mu \mathrm{m})$. The influence of scattering and gas radiation were also investigated. Then, parametric studies of global fluxes emitted by a $1 \mathrm{D}$ slab containing $\mathrm{H}_{2} \mathrm{O}, \mathrm{CO}_{2}$ and particles $(D=1 \mu \mathrm{m})$ at $2000 \mathrm{~K}$ was performed versus the volume fraction and the refractive index. In this particular situation, for all the studied values of the refractive index, the influence of scattering can be neglected $\left(R_{S}<5 \%\right)$ if $f_{v}<6 \times 10^{-6}$ in a $0.1 \mathrm{~m}$ thickness layer or $f_{v}<6 \times 10^{-7}$ in a $1 \mathrm{~m}$ thickness layer. Furthermore, gas radiation can be neglected obviously when absorption by particles is dominant, i.e., when optical index $k$ is around unity $(0.1<k<10)$. In addition, a qualitative approach is proposed to extend the sensitivity results, provided in the paper, to other situations. Finally, the spectral influence of gases and scattering by particles is discussed.

Since this study yields to quantitative results for gases and particles both at thermal equilibrium, the influence of temperature variations can lead to noticeable change in the radiative heat transfer. Another future works of this study address extension to others particle diameters and the development of a validation code which takes into account the scattering processes in a realistic $3 \mathrm{D}$ gas-solid flow.

\section{References}

[1] J.G. Marakis, C. Papapavlou, E. Kakaras, A parametric study of radiative heat transfer in pulverised coal furnaces, Int. J. Heat Mass Transfer 43 (2000) 2961-2971.

[2] W.W. Yuen, L.W. Wong, A parametric study of radiative transfer with anisotropic scattering in a one-dimensional system, J. Quant. Spectrosc. Radiat. Transfer 22 (1979) 231-238.

[3] M.J. Yu, S.W. Baek, J.H. Park, An extension of the weighted sum of gray gases non-gray gas radiation model to a two-phase mixture of non-gray gas with particles, Int. J. Heat Mass Transfer 43 (2000) 1699-1713. 
[4] V. Eymet, R. Fournier, S. Blanco, J.-L. Dufresne, A boundary-based net exchange Monte-Carlo method for absorbing and scattering thick media, J. Quant. Spectrosc. Radiat. Transfer 91 (2005) 27-46.

[5] M. Cherkaoui, J.-L. Dufresne, R. Fournier, J.-Y. Grandpeix, Radiative net exchange formulation within 1D gas enclosures with reflective surfaces, ASME J. Heat Transfer 120 (1998) 275-278.

[6] M. Cherkaoui, J.-L. Dufresne, R. Fournier, J.-Y. Grandpeix, A. Lahellec, Monte-Carlo simulation of radiation in gases with a narrow-band model and a net-exchange formulation, ASME J. Heat Transfer 118 (1996) 401407.

[7] J.-L. Dufresne, R. Fournier, J.-Y. Grandpeix, Méthode de Monte-Carlo par échanges pour le calcul des bilans radiatifs au sein d'une cavité 2D remplie de gaz, Compte-Rendu de l'Académie des Sciences, Paris Série IIb 326 (1998) 33-38.

[8] L. Tessé, F. Dupoirieux, B. Zamuner, J. Taine, Radiative transfer in real gases using reciprocal and forward Monte Carlo methods and a correlated$k$ approach, Int. J. Heat Mass Transfer 3 (2002) 2797-2814.

[9] W.R. Martin, G.C. Pomraning, Monte Carlo analysis of the backscattering of radiation from a sphere to a plane, J. Quant. Spectrosc. Radiat. Transfer 43 (1990) 115-126.

[10] J.-L. Dufresne, R. Fournier, J.-Y. Grandpeix, Inverse Gaussian $k$-distributions, J. Quant. Spectrosc. Radiat. Transfer 61 (1999) 433-441.

[11] A. De Lataillade, J.-L. Dufresne, M. El Hafi, V. Eymet, R. Fournier, A net exchange Monte Carlo approach to radiation in optically thick systems, J. Quant. Spectrosc. Radiat. Transfer 74 (2002) 563-584.

[12] V. Eymet, Simulation Monte-Carlo et analyse en puissances nettes échangées des transferts radiatifs infrarouge avec diffusion : vers une paramétrisation dans un modèle de circulation générale atmosphérique, Ph.D. Thesis, Université Paul Sabatier, http://sphinx-old.ups-tlse.fr/ eymet/, Toulouse, France, 2003.

[13] C. Caliot, Modélisation et simulation de l'émission énergétique et spectrale d'un jet réactif composé de gaz et de particules à haute température issus de la combustion d'un objet pyrotechnique, Ph.D. Thesis, Institut National Polytechnique de Toulouse, http://ethesis.inptoulouse.fr/archive/00000309/, 2006.

[14] J. Taine, A. Soufiani, Gas IR radiative properties: from spectroscopic data to approximate models, Adv. Heat Transfer 33 (1999) 295-341.

[15] S.A. Tashkun, V.I. Perevalov, J.-L. Teffo, A.D. Bykov, N.N. Lavrentieva, CDSD-1000, the high-temperature carbon dioxide spectroscopic databank, J. Quant. Spectrosc. Radiat. Transfer 82 (2003) 165-196.

[16] P. Perez, A. Boischot, L. Ibgui, A. Roblin, A spectroscopic database for water vapor adapted to spectral properties at high temperature, and moderate resolution, J. Quant. Spectrosc. Radiat. Transfer 103 (2007) 231244.

[17] C. Caliot, Y. Le Maoult, M. El Hafi, G. Flamant, Remote sensing of high temperature $\mathrm{H}_{2} \mathrm{O}-\mathrm{CO}_{2}-\mathrm{CO}$ mixture with a correlated $k$-distribution fictitious gas method and the single mixture gas assumption, J. Quant. Spectrosc. Radiat. Transfer 102 (2006) 304-315.

[18] C.F. Bohren, D.R. Huffman, Absorption and Scattering of Light by Small Particles, Wiley, New York, 1983.

[19] R. Siegel, J.R. Howell, Thermal Radiation Heat Transfer, Hemisphere Publishing Corporation, 1992.

[20] G.W. Kattawar, G.N. Plass, Electromagnetic scattering from absorbing spheres, Appl. Opt. 6 (1967) 1377-1383. 